\title{
NONUNIQUENESS OF IMPLICIT LATTICE NAGUMO EQUATION
}

\author{
Petr Stehlík, JonÁš Volek, Plzeň
}

Received October 3, 2018. Published online March 20, 2019.

Abstract. We consider the implicit discretization of Nagumo equation on finite lattices and show that its variational formulation corresponds in various parameter settings to convex, mountain-pass or saddle-point geometries. Consequently, we are able to derive conditions under which the implicit discretization yields multiple solutions. Interestingly, for certain parameters we show nonuniqueness for arbitrarily small discretization steps. Finally, we provide a simple example showing that the nonuniqueness can lead to complex dynamics in which the number of bounded solutions grows exponentially in time iterations, which in turn implies infinite number of global trajectories.

Keywords: reaction-diffusion equation; lattice differential equation; nonlinear algebraic problem; variational method; implicit discretization

MSC 2010: 34A33, 35K57, 39A12, 65Q10

\section{INTRODUCTION}

In this paper we study existence and (non)uniqueness of solutions to the implicit discretization of Nagumo reaction-diffusion equation [17]

$$
u_{t}(x, t)=d u_{x x}(x, t)+r f(u(x, t)), \quad x \in \mathbb{R}, t>0
$$

for various values of the diffusion parameter $d>0$ and reaction parameter $r \in \mathbb{R}$. The Nagumo equation has obtained lot of attention, especially in the case when the nonlinear reaction function $f$ yields two stable states, typically a cubic function $f(u)=u(1-u)(u-a), a \in(0,1)$, or $f(u)=u\left(1-u^{2}\right)$. In such configuration, phase plane analysis provides the existence of traveling wave solutions [10] and the

This publication was supported by the project LO1506 of the Czech Ministry of Education, Youth and Sports under the program NPU I. 
model has been consequently used in many applications, e.g., in the description of propagation of genetic traits [4].

Besides the existence of traveling fronts, it has been shown that the Nagumo equation shows rich behavior with respect to discretization. Spatial discretization of (1.1) yields the Nagumo lattice differential equation (for simplicity of our presentation, we consider only unitary discretization steps in the introduction)

$$
u_{i}^{\prime}(t)=d\left(u_{i-1}(t)-2 u_{i}(t)+u_{i+1}(t)\right)+r f\left(u_{i}(t)\right), \quad i \in \mathbb{Z}, t>0 .
$$

Equation (1.2) not only provides an infinite set of equilibria [14] but also the behaviour of traveling waves is more complicated. Indeed, it has been shown that for sufficiently small diffusion $0<d \ll 1$ the waves connecting the stable states do not travel, the so-called pinning [15], [29].

Furthermore, there is a natural interest in discrete-space models in many applications, e.g., in population dynamics [2], and variants of (1.2) have been studied in higher spatial dimensions to model predator-prey competition [22] and image processing [7]. Similarly, problems generalizing the regular discrete structure, lattice, to general graphs have been considered [24].

Naturally, properties of the fully (and explicitly) discretized equation

$$
u_{i}^{n+1}-u_{i}^{n}=d\left(u_{i-1}^{n}-2 u_{i}^{n}+u_{i+1}^{n}\right)+r f\left(u_{i}^{n}\right), \quad i \in \mathbb{Z}, n \in \mathbb{N}_{0},
$$

have been studied as well, including the number of equilibria, properties of traveling waves [5], [6], maximum principles and their dependence on the temporal discretization [23], [25].

Moreover, numerical schemes for six numerically stable implicit differentiation formulas and their influence on existence and uniqueness of traveling waves have been studied in [13]. Various dynamical properties of abstract implicit difference equations have also been considered [19].

The main goal of this paper is to fully describe the variational structure of the implicit problem on the finite lattice and derive nonuniqueness for several parameter values (motivated by [12], we even briefly consider the problem with negative diffusion $d<0$ ). This finite-dimensional paper follows our infinite-dimensional one [26], in which we were able to describe the geometry of relevant energy functionals in certain cases but only conjecture about the nonuniqueness of solutions (the validity of the Palais-Smale condition remains an open problem and the crucial missing step).

In the case of finite dimensional problem we are not only able to show nonuniqueness of solutions but also to determine exact values of parameters where the geometries of the corresponding energy functional change (see Fig. 3). Moreover, because 
of the finite dimensional lattice, we are able to illustrate the rich structure of solutions. Namely, the number of solutions grows exponentially in time, which leads to infinite number of global trajectories. Interestingly, in certain parameter regions, nonuniqueness is present for arbitrarily small discretization steps.

By considering the variational structure of relevant energy functionals, we can use standard techniques for coercive functionals, mountain-pass or saddle-point geometries. Consequently, our approach is similar to the ideas used in the area of nonlinear algebraic and difference equations [16], [11], [18], [27].

The paper is organized as follows. First, in Section 2 we introduce an initialboundary value problem for the implicit discretization of Nagumo equation on a finite lattice and reformulate it as a variational problem. In Section 3 we show the multiplicity in the bistable case $r>0$. In Section 4 we then extend it also to the monostable case with $r<0$ where the nonuniqueness arises even more naturally. In Section 5 we provide a detailed example for a simple configuration to show the complex dynamics in which the number of bounded solutions grows exponentially in time iterations. Finally, in Section 6 we extend our ideas to the case of negative diffusion $d<0$.

\section{Problem Formulation AND PRELIMINARY CONSIDERATIONS}

Consider the following initial-boundary value problem for reaction-diffusion partial differential equation

$$
\begin{cases}u_{t}(x, t)=d u_{x x}(x, t)+r f(u(x, t)), & x \in(0, l), t \in \mathbb{R}_{0}^{+}, d>0, r \in \mathbb{R}, \\ u(x, 0)=\varphi(x), & x \in(0, l), \\ u(0, t)=0, & t \in \mathbb{R}_{0}^{+} \\ u(l, t)=0, & t \in \mathbb{R}_{0}^{+}\end{cases}
$$

for some initial condition $\varphi$ satisfying $\varphi(0)=\varphi(l)=0$. For the sake of brevity we take into account only the case with the symmetric cubic reaction function $f(u)=$ $u\left(1-u^{2}\right)$. However, most of our ideas can be applied also to the nonsymmetric function $f(u)=u(1-u)(u-a), a \in(0,1)$, which is prevalent in the above mentioned papers. Let us emphasize that we consider the reaction parameter $r$ to be both nonnegative and negative ${ }^{1}$. If $r \geqslant 0$, the reaction term describes the bistable case ${ }^{2}$.

\footnotetext{
${ }^{1}$ Moreover, in Section 6 we also explore the unusual case of negative diffusion $d<0$ (see e.g. [12]).

${ }^{2}$ For the sake of brevity, we include $r=0$ to the bistable case because of the geometry of functionals we consider in this paper. Naturally, the case $r=0$ does not correspond to the bistable case, since the problem (2.1) reduces to a linear equation.
} 
If $r<0$, the reaction function has three zeroes again, however, only the middle one is stable. We call the situation with negative $r$ the monostable case.

We study the implicit discretization of (2.1) obtained by finite differences

$$
\begin{cases}\frac{u_{i}^{n+1}-u_{i}^{n}}{h}=d \frac{u_{i-1}^{n+1}-2 u_{i}^{n+1}+u_{i+1}^{n+1}}{\mu^{2}}+r u_{i}^{n+1}\left(1-\left(u_{i}^{n+1}\right)^{2}\right), & i \in\{1,2, \ldots, N\}, n \in \mathbb{N}_{0}, \\ u_{i}^{0}=\varphi_{i}, & i \in\{1,2, \ldots, N\}, \\ u_{0}^{n}=0, & n \in \mathbb{N}_{0}, \\ u_{N+1}^{n}=0, & n \in \mathbb{N}_{0},\end{cases}
$$

in which we denote by $\mu=l /(N+1)$ a spatial discretization step and by $h>0$ a temporal discretization step.

The implicit discrete problem (2.2) can be reformulated into an algebraic form. We introduce the following additional notation. Let $L \in \mathbb{R}^{N \times N}$ and $S: \mathbb{R}^{N} \rightarrow \mathbb{R}^{N}$ be a continuous mapping defined as follows,

$$
L=\left(\begin{array}{cccccc}
-2 & 1 & 0 & & 0 & 0 \\
1 & -2 & 1 & \ldots & 0 & 0 \\
0 & 1 & -2 & & 0 & 0 \\
& \vdots & & \ddots & & \\
0 & 0 & 0 & & 1 & -2
\end{array}\right), \quad S(u)=\left(\begin{array}{c}
u_{1}\left(1-\left(u_{1}\right)^{2}\right) \\
u_{2}\left(1-\left(u_{2}\right)^{2}\right) \\
\vdots \\
u_{N}\left(1-\left(u_{N}\right)^{2}\right)
\end{array}\right)
$$

The tridiagonal matrix $L$ is negative definite and has the strictly decreasing sequence of eigenvalues (see e.g. [1], Section 5.3.3 for a detailed derivation)

$$
\nu_{k}=-2\left(1-\cos \frac{k \pi}{N+1}\right), \quad k=1,2, \ldots, N,
$$

with the corresponding system of orthogonal eigenvectors

$$
\eta_{k}=\left(\sin \frac{k \pi}{N+1}, \sin \frac{2 k \pi}{N+1}, \ldots, \sin \frac{N k \pi}{N+1}\right)^{\top}, \quad k=1,2, \ldots, N .
$$

Straightforwardly, we have $\nu_{k} \in(-4,0)$ for every $k=1,2, \ldots, N$.

Furthermore, let $n \in \mathbb{N}_{0}$ and $\left(u_{i}^{n}\right)_{i=0}^{N+1}$ be given and $\left(u_{i}^{n+1}\right)_{i=0}^{N+1}$ unknown. Denote

$$
b=\left(u_{1}^{n}, u_{2}^{n}, \ldots, u_{N}^{n}\right)^{\top}, \quad u=\left(u_{1}^{n+1}, u_{2}^{n+1}, \ldots, u_{N}^{n+1}\right)^{\top} .
$$

Under the notation (2.3)-(2.6) and for a fixed $\left(u_{i}^{n}\right)_{i=0}^{N+1}$ one can rewrite the problem of finding $\left(u_{i}^{n+1}\right)_{i=0}^{N+1}$ satisfying (2.2) into the following fixed-point problem:

$$
u=b+\frac{h d}{\mu^{2}} L u+h r S(u), \quad u \in \mathbb{R}^{N} .
$$


Thanks to the symmetry of the matrix $L$ one can find the energy functional $F$ : $\mathbb{R}^{N} \rightarrow \mathbb{R}$ associated to $(2.7)$ as

$$
F(u)=\frac{1-h r}{2}\|u\|_{2}^{2}-(b, u)-\frac{h d}{2 \mu^{2}}(L u, u)+\frac{h r}{4}\|u\|_{4}^{4}
$$

in which $(u, v)=\sum_{i=1}^{N} u_{i} v_{i}$ is the scalar product, $\|u\|_{2}=\sqrt{(u, u)}$ is the Euclidean norm, and

$$
\|u\|_{4}=\sqrt[4]{\sum_{i=1}^{N}\left(u_{i}\right)^{4}}
$$

is the 4 -norm in $\mathbb{R}^{N}$.

The following lemma is an immediate consequence of the fact that $L, S$ are continuous mappings.

Lemma 2.1. The functional $F$ given by $(2.8)$ satisfies $F \in C^{1}\left(\mathbb{R}^{N}, \mathbb{R}\right)$. Moreover, $u \in \mathbb{R}^{N}$ is a critical point of $F$ (i.e., $\nabla F(u)=0$ ) if and only if it is a solution of (2.7).

P r o of. See [26], Lemma 3.1, Lemma 3.5 for a similar proof.

Lemma 2.2. Let $b_{1}, b_{2} \in \mathbb{R}^{N}, b_{1} \neq b_{2}$, and let $F_{1}, F_{2}$ be corresponding functionals defined by (2.8) with $b=b_{1}, b=b_{2}$, respectively. Let $u_{1} \in \mathbb{R}^{N}$ a critical point of $F_{1}$ and $u_{2} \in \mathbb{R}^{N}$ a critical point of $F_{2}$. Then $u_{1} \neq u_{2}$.

Pro of. Suppose that $u_{1}=u_{2}=u$. Then

$$
\nabla F_{1}(u)=u-b_{1}-\frac{h d}{\mu^{2}} L u-h r S(u)=0=u-b_{2}-\frac{h d}{\mu^{2}} L u-h r S(u)=\nabla F_{2}(u),
$$

which implies $b_{1}=b_{2}$, a contradiction.

In the following sections we study the existence and (non)uniqueness of solutions of the implicit discrete problem (2.2) applying the above stated reformulation and the variational analysis of the energy functional $F$. We distinguish between two cases, when the reaction term is bistable $(r \geqslant 0)$, and monostable $(r<0)$.

Nonetheless, before we analyze the geometry of $F$ in various parameter configurations, let us state one general property. Since the form of the functional $F$ (2.8) can be rewritten into

$$
F(u)=\left(\left(\frac{1-h r}{2} I-\frac{h d}{2 \mu^{2}} L\right) u, u\right)-(b, u)+\frac{h r}{4}\|u\|_{4}^{4}
$$


( $I$ denotes the identity matrix) one can see that the geometry strongly depends on the definiteness of the matrix

$$
A=\frac{1-h r}{2} I-\frac{h d}{2 \mu^{2}} L
$$

We denote by $\lambda_{1} \leqslant \lambda_{2} \leqslant \ldots \leqslant \lambda_{N}$ the eigenvalues of $A$. It can be shown from (2.4) that the eigenvalues $\lambda_{k}, k=1,2, \ldots, N$, form a strictly increasing sequence

$$
\lambda_{k}=\frac{1-h r}{2}-\frac{h d}{2 \mu^{2}} \nu_{k}, \quad k=1,2, \ldots, N
$$

with the system of eigenvectors $\phi_{k}, k=1,2, \ldots, N$, which is completely the same as $(2.5)$, i.e. $\phi_{k}=\eta_{k}, k=1,2, \ldots, N$. We have immediately

$$
\lambda_{1}\|u\|_{2}^{2} \leqslant\left(\left(\frac{1-h r}{2} I-\frac{h d}{2 \mu^{2}} L\right) u, u\right) \leqslant \lambda_{N}\|u\|_{2}^{2} \quad \forall u \in \mathbb{R}^{N}
$$

\section{Bistable REACTION TERM}

In this section we consider $r \geqslant 0$, i.e., the reaction term in (2.2) being bistable. The following lemma summarizes the elementary properties of the functional $F$ provided $r \geqslant 0$.

Lemma 3.1. Let $r \geqslant 0$. Then for the functional $F$ given by $(2.8)$ the following assertions hold:

(i) $F$ is weakly coercive, i.e., $F(u) \rightarrow \infty$ for $\|u\|_{2} \rightarrow \infty$,

(ii) $F$ is bounded from below,

(iii) F satisfies the Palais-Smale condition:

(PS) Every sequence $\left\{u_{n}\right\} \subset \mathbb{R}^{N}$ such that $\left\{F\left(u_{n}\right)\right\} \subset \mathbb{R}$ is bounded and $\left\|\nabla F\left(u_{n}\right)\right\|_{2} \rightarrow 0$ possesses a convergent subsequence.

Pr o of. First, since we work in the space $\mathbb{R}^{N}$, we have

$$
\|u\|_{4} \leqslant\|u\|_{2} \leqslant N^{\frac{1}{4}}\|u\|_{4} \quad \forall u \in \mathbb{R}^{N} .
$$

To prove (i), let $\|u\|_{2} \rightarrow \infty$. One can obtain by (2.11), the Cauchy-Schwarz inequality, and (3.1) that

$$
F(u) \geqslant \lambda_{1}\|u\|_{2}^{2}-\|b\|_{2}\|u\|_{2}+\frac{h r}{4 N}\|u\|_{2}^{4} \rightarrow \infty .
$$


Indeed, if $r=0$, then $\lambda_{1}>0$ by (2.10) and $\|u\|_{2}^{2} \gg\|u\|_{2}$ for $\|u\|_{2} \rightarrow \infty$. If $r>0$, then $\|u\|_{2}^{4} \gg\|u\|_{2}^{2}$ for $\|u\|_{2} \rightarrow \infty$.

The statements (ii) and (iii) are immediate consequences of the weak coercivity of $F$. Indeed, let $\left\{u_{n}\right\} \subset \mathbb{R}^{N}$ be such that $F\left(u_{n}\right) \rightarrow-\infty$. Then the weak coercivity of $F$ yields immediately that $\left\|u_{n}\right\|_{2}$ is bounded. Since we are in a finite dimension, there has to be $u_{n} \rightarrow u_{0}$ (at least for a subsequence). Consequently, $F\left(u_{n}\right) \rightarrow$ $F\left(u_{0}\right)>-\infty$ because $F \in C^{1}\left(\mathbb{R}^{N}, \mathbb{R}\right)$, a contradiction. The statement (iii) can be obtained in a similar way.

Lemma 3.1 immediately implies the existence of at least one global solution of $(2.2)$.

Theorem 3.2. Let $r \geqslant 0$. Then the problem (2.2) has at least one solution $u$ which exists for every $(n, i) \in \mathbb{N}_{0} \times\{0,1,2, \ldots, N, N+1\}$.

Pr o of. We proceed via mathematical induction on $n \in \mathbb{N}_{0}$. For $n=0$ we have $u_{0}^{0}=u_{N+1}^{0}=0, u_{i}^{0}=\varphi_{i}, i=1,2, \ldots, N$. Let $n \in \mathbb{N}_{0}$ and $\left(u_{i}^{n}\right)_{i=0}^{N+1}$ be given. Then using the variational reformulation from Section 2 , there exists a solution $\left(u_{i}^{n+1}\right)_{i=0}^{N+1}$ at $n+1$ if and only if the corresponding $u=\left(u_{1}^{n+1}, u_{2}^{n+1}, \ldots, u_{N}^{n+1}\right)^{\top}$ is a critical point of $F$. Since $F$ is bounded from below and satisfies (PS) by Lemma 3.1, it has at least one critical point which is a global minimizer (see e.g. [21], Theorem 2.7). Consequently, there exists at least one solution at $n+1$.

The (non)uniqueness of solutions of (2.2) depends on a specific geometry of the energy functional $F$. However, as we mentioned above, the geometry strongly depends on the definiteness of the matrix $A=\frac{1}{2}(1-h r) I-\left(\frac{1}{2} h d / \mu^{2}\right) L$, determined by the parameters $r, d, h$, and $\mu$. Therefore, we distinguish several cases. First, we discuss the problem with $A$ being positive (semi)definite.

Theorem 3.3. Let $r \geqslant 0$ and $\lambda_{1} \geqslant 0$. Then the solution $u$ from Theorem 3.2 is unique.

P r o of. Let $n \in \mathbb{N}_{0}$ be given and let $\left(u_{i}^{n}\right)_{i=0}^{N+1}$ be uniquely determined. Suppose that there exist two distinct solutions $\left(u_{i}^{n+1}\right)_{i=0}^{N+1},\left(v_{i}^{n+1}\right)_{i=0}^{N+1}$ at $n+1$. This yields that $u=\left(u_{1}^{n+1}, u_{2}^{n+1}, \ldots, u_{N}^{n+1}\right)^{\top}$ and $v=\left(v_{1}^{n+1}, v_{2}^{n+1}, \ldots, v_{N}^{n+1}\right)^{\top}$ are distinct critical points of the functional $F$. However, $F$ is strictly convex due to the assumptions that $r \geqslant 0$ and $\lambda_{1} \geqslant 0$. Indeed, $F$ satisfies $F=F_{1}+F_{2}+F_{3}$ in which

$$
\begin{aligned}
& \left.F_{1}(u)=\left(\left(\frac{1-h r}{2} I-\frac{h d}{2 \mu^{2}} L\right) u, u\right) \text { is convex (strictly convex for } \lambda_{1}>0\right), \\
& F_{2}(u)=-(b, u) \text { is linear and thus convex, } \\
& \left.F_{3}(u)=\frac{h r}{4}\|u\|_{4}^{4} \text { is convex (strictly convex for } \lambda_{1}=0, \text { which implies } r>0\right) .
\end{aligned}
$$


Therefore, $F$ has a unique critical point which is a global minimizer (see e.g. [9], Proposition 7.1.8, Theorem 7.2.12), a contradiction.

The formula (2.10) and Theorem 3.3 immediately imply the following statement.

Corollary 3.4. Let $0 \leqslant r \leqslant 1 / h-d \nu_{1} / \mu^{2}$. Then the solution $u$ from Theorem 3.2 is unique.

We turn our attention now to the case in which $A=\frac{1}{2}(1-h r) I-\left(\frac{1}{2} h d / \mu^{2}\right) L$ is negative definite. In this situation we expect a mountain pass geometry of the functional $F$ and thus, more than one critical point of $F$.

Before we start this analysis, we introduce some auxiliary notation and considerations. Let $g: \mathbb{R}_{0}^{+} \rightarrow \mathbb{R}$ be a real function defined as

$$
g(\varrho)=\alpha \varrho-\beta \varrho^{3}, \quad \alpha, \beta>0
$$

One can directly show that this function is strictly concave (note that $\varrho \geqslant 0$ ). It has two zeroes at $\varrho=0$ and $\varrho=\sqrt{\alpha / \beta}$. It has a global maximum at $\varrho_{m}=\sqrt{\alpha /(3 \beta)}$ with $g\left(\varrho_{m}\right)=\max _{\varrho \in \mathbb{R}_{0}^{+}} g(\varrho)=\frac{2}{3} \alpha \sqrt{\alpha /(3 \beta)}, g$ is strictly increasing on $\left[0, \varrho_{m}\right]$, and strictly decreasing on $\left[\varrho_{m}, \infty\right)$.

Suppose that $\lambda_{N}<0\left(A=\frac{1}{2}(1-h r) I-\left(\frac{1}{2} h d / \mu^{2}\right) L\right.$ is negative definite $)$ which immediately implies that $r>0$.

Lemma 3.5. Let $\lambda_{N}<0$ and $\|b\|_{2}<-\frac{2}{3} \lambda_{N} \sqrt{-4 \lambda_{N} /(3 h r)}$. Then the functional $F$ given by (2.8) has at least three critical points. Moreover, if $\lambda_{N} \leqslant-\frac{3}{2}$, there exists a critical point $u_{M} \in \mathbb{R}^{N}$ for which $\left\|u_{M}\right\|_{2}<-\frac{2}{3} \lambda_{N} \sqrt{-4 \lambda_{N} /(3 h r)}$.

Proof. Let $\|u\|_{2}=\varrho$. We obtain the following estimate using (2.11), the Cauchy-Schwarz inequality, and (3.1):

$$
F(u) \leqslant \lambda_{N}\|u\|_{2}^{2}+\|b\|_{2}\|u\|_{2}+\frac{h r}{4}\|u\|_{2}^{4}=-\varrho\left(\alpha \varrho-\beta \varrho^{3}-\|b\|_{2}\right),
$$

where $\alpha=-\lambda_{N}>0$ and $\beta=\frac{1}{4} h r>0$. We want to show that there exists $\varrho_{0}>0$ such that $F(u)<0$ for all $\|u\|_{2}=\varrho_{0}$. According to (3.3) it is sufficient to find $\varrho_{0}>0$ such that

$$
g\left(\varrho_{0}\right)=\alpha \varrho_{0}-\beta \varrho_{0}>\|b\|_{2} .
$$

Since

$$
\|b\|_{2}<-\frac{2}{3} \lambda_{N} \sqrt{-\frac{4 \lambda_{N}}{3 h r}}=\frac{2}{3} \alpha \sqrt{\frac{\alpha}{3 \beta}}=g\left(\varrho_{m}\right)=\max _{\varrho \in \mathbb{R}_{0}^{+}} g(\varrho),
$$


putting $\varrho_{0}=\varrho_{m}$ the inequality (3.4) is satisfied and thus, $F(u) \leqslant \max _{\|u\|=\varrho_{0}} F(u)<0$ for all $\|u\|_{2}=\varrho_{0}$.

Consider the functional $G=-F$. Since $G(0)=0$ and $G(u) \geqslant \min _{\|u\|=\varrho_{0}} G(u)>0$ for $\|u\|=\varrho_{0}$ by $(3.3)$ and (3.4), there exists a local minimizer $u_{M}$ of $G$ such that $G\left(u_{M}\right)=\min _{\|u\|_{2} \leqslant \varrho_{0}} G(u) \leqslant 0\left(G \in C^{1}\left(\mathbb{R}^{N}, \mathbb{R}\right)\right.$ and we work in a finite dimension $)$. Further, Lemma 3.1 yields that $G$ is bounded from above and satisfies (PS). Thus, the direct maximization (see e.g. [21], Theorem. 2.7) yields that there exists a global maximizer $u_{X}$ of $G$ such that $G\left(u_{X}\right)>0$, i.e. $u_{X} \neq u_{M}$.

Lemma 3.1 yields also that $F$ is weakly coercive, which implies that there exists $e$ such that $\|e\|_{2}>\varrho_{0}$ and $G(e) \leqslant G(0)=0$. Again, we have $G(u) \geqslant \min _{\|u\|=\varrho_{0}} G(u)>0$ for $\|u\|=\varrho_{0}$ by (3.3) and (3.4). Therefore, the Mountain Pass Theorem (see [3], or the survey paper [21], Theorem 2.2) implies that there is a critical point $u_{S}$ of $G$ such that

$$
\begin{aligned}
G\left(u_{S}\right)=\inf _{\gamma \in \Gamma} \max _{t \in[0,1]} G(\gamma(t))>0, \\
\qquad \text { with } \Gamma=\left\{\gamma \in C\left([0,1], \mathbb{R}^{N}\right): \gamma(0)=0, \gamma(1)=e\right\}
\end{aligned}
$$

We have to additionally discuss the possibility of $u_{S}=u_{X}$. Hence, suppose that $u_{S}=u_{X}$. Let $\gamma_{0}$ be a curve from $\Gamma$ such that $u_{S}=u_{X} \notin \gamma_{0}([0,1])$. Since $G\left(u_{X}\right)=$ $\max _{u \in \mathbb{R}^{N}} G(u)$, we have $G(u) \leqslant G\left(u_{S}\right)$ for all $u \in \gamma_{0}([0,1])$. If there is a $u_{P} \in \gamma_{0}([0,1])$ such that $G\left(u_{P}\right)=G\left(u_{X}\right)$, it is a second global maximizer of $G$, i.e., a third critical point. Otherwise, if $G(u)<G\left(u_{X}\right)$ for all $u \in \gamma_{0}([0,1])$, we obtain a contradiction with a characterization of $u_{S}$ by $(3.5)$.

Consequently, $G$ has at least three critical points which are obviously critical points of $F$ as well.

Finally, we have $\left\|u_{M}\right\|_{2}<\varrho_{0}$. The additional assumption $-\alpha=\lambda_{N} \leqslant-\frac{3}{2}$ yields that $\varrho_{0}=\sqrt{\alpha /(3 \beta)} \leqslant \frac{2}{3} \alpha \sqrt{\alpha /(3 \beta)}$. Hence, we have $\left\|u_{M}\right\|_{2}<\varrho_{0} \leqslant \frac{2}{3} \alpha \sqrt{\alpha /(3 \beta)}=$ $-\frac{2}{3} \lambda_{N} \sqrt{-4 \lambda_{N} /(3 h r)}$.

Now we can apply Lemma 3.5 to prove the following two nonuniqueness results.

Theorem 3.6. Let $\lambda_{N}<0$ and $\varphi=\left(\varphi_{1}, \varphi_{2}, \ldots, \varphi_{N}\right)^{\top}$ be such that $\|\varphi\|_{2}<$ $-\frac{2}{3} \lambda_{N} \sqrt{-4 \lambda_{N} /(3 h r)}$. Then at each time instant $n \in \mathbb{N}$ there exist at least three solutions of (2.2) which form trajectories $u^{n}, n \rightarrow \infty$.

Pr o of. Let $b=\varphi$. Lemma 3.5 implies that there exist at least three critical points of $F$. Hence, there are at least three solutions of $(2.2)$ at $n=1$. Please note that if we put one of these solutions as a new $b$ to continue inductively, it need not be $\|b\|_{2}<-\frac{2}{3} \lambda_{N} \sqrt{-4 \lambda_{N} /(3 h r)}$ and we cannot apply Lemma 3.5 again. 
Analogously as in the proof of Theorem 3.2, $F$ is bounded from below and satisfies (PS) by Lemma 3.1 (independently of the value of $\|b\|_{2}$ ). This yields that there is always a global minimizer of $F$ (see e.g. [21], Theorem 2.7 again). This means that each of those three solutions at $n=1$ continues for higher values of $n$. Moreover, these three global solutions cannot coincide by Lemma 2.2 .

Theorem 3.7. Let $\lambda_{N} \leqslant-\frac{3}{2}$ and $\|\varphi\|_{2}<-\frac{2}{3} \lambda_{N} \sqrt{-4 \lambda_{N} /(3 h r)}$. Then at each time instant $n \in \mathbb{N}$ there are at least $2 n+1$ solutions of (2.2) which form trajectories $u^{n}, n \rightarrow \infty$.

Proof. We prove the statement by mathematical induction. For $n=1$ we obtain as in the proof of Theorem 3.6 at least three solutions of (2.2) which correspond to critical points $u_{M}, u_{X}, u_{S}$ of $F$. The assumption $\lambda_{N}<-\frac{3}{2}$ implies moreover that $\left\|u_{M}\right\|_{2}<-\frac{2}{3} \lambda_{N} \sqrt{-4 \lambda_{N} /(3 h r)}$.

Now we prove the induction step. Let $n \in \mathbb{N}$ be given. Then there exist at least $2 n+1$ solutions of (2.2) at $n$ and one of them, say $\left(\bar{u}_{i}^{n}\right)_{i=0}^{N+1}$, corresponds to the critical point $u_{M}$ of $F$ with an appropriate $b$, and $\left\|u_{M}\right\|_{2}<-\frac{2}{3} \lambda_{N} \sqrt{-4 \lambda_{N} /(3 h r)}$. Redefining $b=\bar{u}$, in which $\bar{u}=\left(\bar{u}_{1}^{n}, \bar{u}_{2}^{n}, \ldots, \bar{u}_{N}^{n}\right)^{\top}$, Lemma 3.5 yields that the functional $F$ has again three critical points $u_{M}, u_{X}, u_{S}$ with $\left\|u_{M}\right\|_{2}<-\frac{2}{3} \lambda_{N} \sqrt{-4 \lambda_{N} /(3 h r)}$ which form solutions of (2.2) at $n+1$. Further, the other $2 n$ solutions at $n$ continues by at least one solution at $n+1$ as in the proof of Theorem 3.6 again. Lemma 2.2 yields that all these solutions cannot coincide. Therefore, we have $3+2 n=2(n+1)+1$ solutions of (2.2) at $n+1$. The induction step is complete.

The formula (2.10) in combination with Theorems 3.6 and 3.7 yield the following statement.

Corollary 3.8. Let $r>1 / h-d \nu_{N} / \mu^{2}$ and

$$
\|\varphi\|_{2}<\frac{1}{3 \mu^{2}}\left(h \mu^{2} r-\mu^{2}+h d \nu_{N}\right) \sqrt{\frac{2}{3 h \mu^{2} r}\left(h \mu^{2} r-\mu^{2}+h d \nu_{N}\right)} .
$$

Then at each time instant $n \in \mathbb{N}$ there exist at least three solutions of (2.2) which form trajectories $u^{n}, n \rightarrow \infty$. Moreover, if $r \geqslant 4 / h-d \nu_{N} / \mu^{2}$, then at each time instant $n \in \mathbb{N}_{0}$ there exist at least $2 n+1$ solutions of (2.2) which form trajectories $u^{n}, n \rightarrow \infty$.

R e m a r k 3.9. We note:

(i) We expect that the number of solutions of (2.2) can depend even exponentially on $n$ in the nonuniqueness case (actually, independently of the sign of $r$ ). We illustrate this phenomenon by a simple example in Section 5 . 
(ii) We expect that the number of solutions of (2.2) depends also on the dimension $N$, i.e., on the number of points in the spatial lattice. For example, suppose that $\varphi_{i}=0$ for all $i \in\{1,2, \ldots, N\}$. Then the solutions at $n=1$ correspond to critical points of $F$ with $b=0$. In this case $F$ is an even functional and one can apply Clark's theorem (see [8], or a survey paper [21], Theorem 9.1) under the assumption that $\lambda_{N}<0$ to prove that $F$ has at least $N$ distinct pairs of critical points. Thus, there exist at least $2 N+1$ solutions at $n=1$. However, in this case we cannot proceed by induction, because we lost the evenness of the functional $F$ for solutions at $n=2$.

At the end of this section we would like to briefly introduce the most complicated case in which the matrix $A=\frac{1}{2}(1-h r) I-\left(\frac{1}{2} h d / \mu^{2}\right) L$ is indefinite (or negative semidefinite).

Lemma 3.10. Let $\lambda_{1}<\ldots<\lambda_{j}<0 \leqslant \lambda_{j+1}<\ldots<\lambda_{N}$. Then there exists $\delta>0$ such that for every $b \in \mathbb{R}^{N}$ satisfying $\|b\|_{2}<\delta$ the functional $F$ given by (2.8) has at least three critical points.

Pr o of. The statement follows from [28], Theorem 1.1. This theorem states the following. Consider a continuously differentiable functional on a Banach space $X$ which is bounded from below, satisfies (PS) and the geometrical assumption of the Saddle Point Theorem (see [20], or the survey paper [21], Theorem 4.6), specifically, there is $X=Y \oplus Z$, with $1 \leqslant \operatorname{dim} Y<\infty$, such that there exists $\varrho_{0}>0$ for which

$$
\max _{u \in Y,\|u\|=\varrho_{0}} F(u)<\inf _{u \in Z} F(u)
$$

Then there exists at least three critical points of such a functional.

Lemma 3.1 yields that the functional $F$ given by (2.8) is bounded from below and satisfies (PS). Thus, in the rest of the proof we verify the assumption (3.6) for a suitable choice of $Y, Z$, and $\varrho_{0}$.

Define $Y=\operatorname{span}\left(\phi_{1}, \phi_{2}, \ldots, \phi_{j}\right)$, and $Z=\operatorname{span}\left(\phi_{j+1}, \phi_{j+2}, \ldots, \phi_{N}\right)$, where $\phi_{k}$ denotes an eigenvector corresponding to $\lambda_{k}$, i.e., $\left\{\phi_{k}\right\}_{k=1}^{N}$ forms an orthogonal basis of $\mathbb{R}^{N}$. Obviously, $\mathbb{R}^{N}=Y \oplus Z$.

Let $u \in Z$. Since $\lambda_{k} \geqslant 0$ for $k \geqslant j+1$, we have

$$
\left(\left(\frac{1-h r}{2} I-\frac{h d}{2 \mu^{2}} L\right) u, u\right) \geqslant 0 \text {. }
$$

Therefore, we obtain by the Cauchy-Schwarz inequality and (3.1) that for all $u \in Z$ we have

$$
F(u) \geqslant-\|b\|_{2}\|u\|_{2}+\frac{h r}{4 N}\|u\|_{2}^{4}
$$


One can easily verify that

$$
\min _{\varrho \geqslant 0}\left(-\|b\|_{2} \varrho+\frac{h r}{4 N} \varrho^{4}\right)=-\frac{3}{4}\|b\|_{2} \sqrt[3]{\frac{N\left\|^{2}\right\|_{2}}{h r}} .
$$

Hence, (3.7) yields that

$$
\inf _{u \in Z} F(u) \geqslant-\frac{3}{4}\|b\|_{2} \sqrt[3]{\frac{N\|b\|_{2}}{h r}} .
$$

Let $u \in Y$ and $u=\sum_{k=1}^{j} a_{k} \phi_{k}$. Then

$$
\left(\left(\frac{1-h r}{2} I-\frac{h d}{2 \mu^{2}} L\right) u, u\right)=\sum_{k=1}^{j} \lambda_{k} a_{k}^{2} \leqslant \lambda_{j}\|u\|_{2}^{2} .
$$

Therefore, for all $u \in Y$,

$$
F(u) \leqslant \lambda_{j}\|u\|_{2}^{2}+\|b\|_{2}\|u\|_{2}+\frac{h r}{4}\|u\|_{2}^{4} .
$$

Consequently, to satisfy (3.6) we have to find $\varrho_{0}>0$ such that

$$
\lambda_{j} \varrho_{0}^{2}+\|b\|_{2} \varrho_{0}+\frac{h r}{4} \varrho_{0}^{4}<-\frac{3}{4}\|b\|_{2} \sqrt[3]{\frac{N\|b\|_{2}}{h r}} .
$$

We rewrite the inequality (3.8) into the following form for the variable $\varrho \geqslant 0$ :

$$
\lambda_{j} \varrho^{2}+\frac{h r}{4} \varrho^{4}<-\|b\|_{2} \varrho-\frac{3}{4}\|b\|_{2} \sqrt[3]{\frac{N\|b\|_{2}}{h r}} .
$$

One can see that the left-hand side of (3.9) is a function of $\varrho \geqslant 0$ which is independent of $\|b\|_{2}$. Since $\lambda_{j}<0$, the left-hand side of (3.9) has a negative global minimum at some $\varrho_{0}>0$. The value of that negative minimum and the value $\varrho_{0}>0$ are independent of $\|b\|_{2}$ as well. Since the value

$$
-\|b\|_{2} \varrho_{0}-\frac{3}{4}\|b\|_{2} \sqrt[3]{\frac{N\|b\|_{2}}{h r}}
$$

on the right-hand side of (3.9) converges to zero for $\|b\|_{2} \rightarrow 0$, there has to exist $\delta>0$ such that for $\|b\|_{2}<\delta$ we have

$$
\lambda_{j} \varrho_{0}^{2}+\frac{h r}{4} \varrho_{0}^{4}<-\|b\|_{2} \varrho_{0}-\frac{3}{4}\|b\|_{2} \sqrt[3]{\frac{N\|b\|_{2}}{h r}} .
$$

Hence, the inequality (3.6) is satisfied for $u \in Y,\|u\|_{2}=\varrho_{0}$, and $\|b\|_{2}<\delta$.

Since all the assumptions of [28], Theorem 1.1 are satisfied, there exist at least three critical points of the functional $F$ provided $\|b\|_{2}<\delta$. 
Theorem 3.11. Let $\lambda_{1}<\ldots<\lambda_{j}<0 \leqslant \lambda_{j+1}<\ldots<\lambda_{N}$ and $\delta>0$ be from Lemma 3.10. If $\|\varphi\|_{2}<\delta$ ( $\|\varphi\|_{2}$ is sufficiently small), then at each time instant $n \in \mathbb{N}$ there are at least three solutions of (2.2) which form trajectories $u^{n}, n \rightarrow \infty$.

Pr o of. The proof can be done in a way similar to the proof of Theorem 3.6 applying Lemma 3.10 .

R e m a r $\mathrm{k}$ 3.12. We cannot show the existence of at least $2 n+1$ solutions of $(2.2)$ at time instant $n \in \mathbb{N}_{0}$ for the case with an indefinite matrix $A=\frac{1}{2}(1-h r) I-$ $\left(\frac{1}{2} h d / \mu^{2}\right) L$ as in Theorem 3.7. There is no easy a priori bound on the value $\delta$ and on the norms of critical points of $F$. Thus, we cannot apply mathematical induction with the saddle geometry of $F$.

The results of this section and the consequent dependence of the number of solutions on the eigenvalues of the matrix $A=\frac{1}{2}(1-h r) I-\left(\frac{1}{2} h d / \mu^{2}\right) L$ and the reaction parameter $r$ are depicted in Figs. 1 and 3.

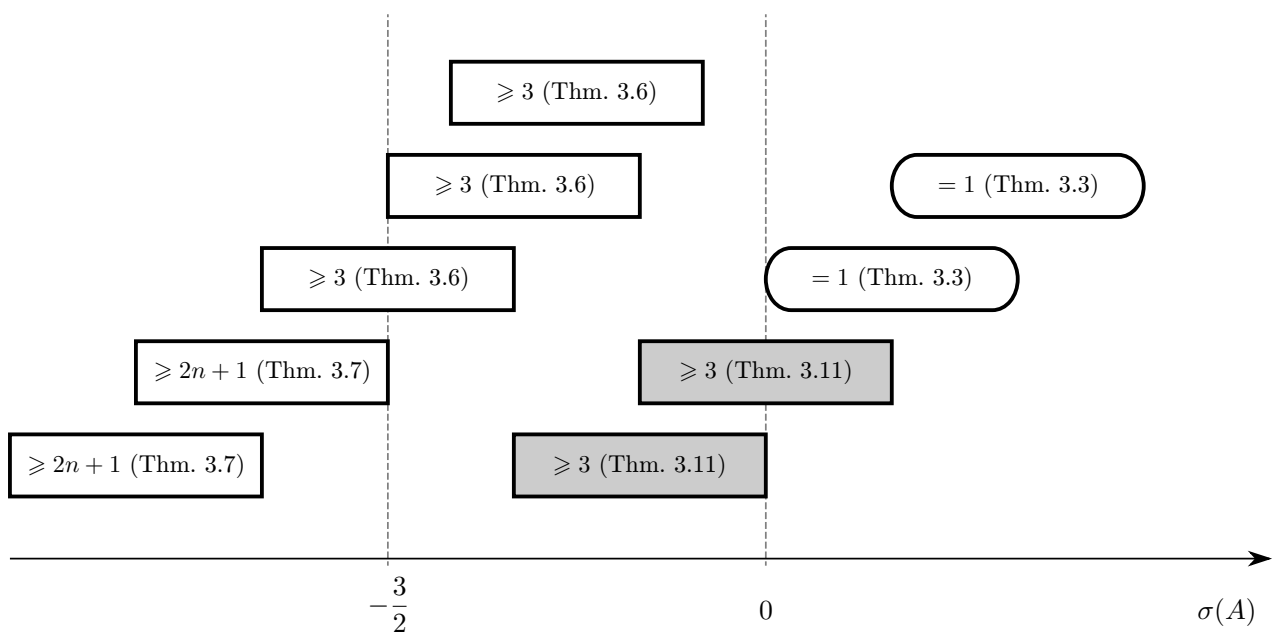

Figure 1. Dependence of the number of solutions in the bistable case $r \geqslant 0$ with positive diffusion based on the spectrum $\sigma(A)$ of the matrix $A=\frac{1}{2}(1-h r) I-\left(\frac{1}{2} h d / \mu^{2}\right) L$. The picture depicts various positions of $\lambda_{1}$ (the left end of the region) and $\lambda_{N}$ (the right end). A rectangle corresponds to the mountain-pass geometry, a shaded rectangle to the saddle geometry and the rounded rectangle to the convex geometry. 


\section{Monostable reaction term}

In this section we analyze the existence and (non)uniqueness of solutions to (2.2) assuming that the reaction parameter $r$ is strictly negative and thus, the reaction term is monostable. Thus, suppose that $r<0$ throughout this section.

First, we emphasize that for $r<0$ we have $\lambda_{1}>0$ by (2.10) for all values of $d, h$, and $\mu$ and thus, $A=\frac{1}{2}(1-h r) I-\left(\frac{1}{2} h d / \mu^{2}\right) L$ is always positively definite. However, contrary to the bistable case, we have $\frac{1}{4} h r\|u\|_{2}^{4} \rightarrow-\infty$ for $\|u\|_{2} \rightarrow \infty$. Consequently, if the reaction term is monostable, the energy functional $F$ has a mountain pass geometry independently of the values of $r, d, h$, and $\mu$ and we expect nonuniqueness whenever $r<0$.

Lemma 4.1. Let $r<0$. Then for the functional $F$ given by $(2.8)$ the following assertions hold:

(i) $F(u) \rightarrow-\infty$ for $\|u\|_{2} \rightarrow \infty$,

(ii) $F$ is bounded from above,

(iii) $F$ satisfies (PS).

Proof. Analogously as in the proof of Lemma 3.1 it is sufficient to prove (i), since (ii) and (iii) are its immediate consequences. Therefore, let $\|u\|_{2} \rightarrow \infty$. By (2.11), the Cauchy-Schwarz inequality, and (3.1) we get

$$
F(u) \leqslant \lambda_{N}\|u\|_{2}^{2}+\|b\|_{2}\|u\|_{2}+\frac{h r}{4 N}\|u\|_{2}^{4} \rightarrow-\infty,
$$

since $r<0$ and $\|u\|_{2}^{4} \gg\|u\|_{2}^{2}$ for $\|u\|_{2} \rightarrow \infty$.

Lemma 4.2. Let $r<0$ and $\|b\|_{2}<\frac{2}{3} \lambda_{1} \sqrt{-4 \lambda_{1} /(3 h r)}$. Then the functional $F$ given by (2.8) has at least three critical points. Moreover, if $\lambda_{1} \geqslant \frac{3}{2}$, there exists a critical point $u_{M} \in \mathbb{R}^{N}$ for which $\left\|u_{M}\right\|_{2}<\frac{2}{3} \lambda_{1} \sqrt{-4 \lambda_{1} /(3 h r)}$.

Pr o of. The proof follows steps similar to the proof of Lemma 3.5. Let $\|u\|_{2}=\varrho$. Applying (2.11), the Cauchy-Schwarz inequality, and (3.1), we obtain

$$
F(u) \geqslant \lambda_{1}\|u\|_{2}^{2}-\|b\|_{2}\|u\|_{2}+\frac{h r}{4}\|u\|_{2}^{4}=\varrho\left(\alpha \varrho-\beta \varrho^{3}-\|b\|_{2}\right),
$$

in which $\alpha=\lambda_{1}>0, \beta=-\frac{1}{4} h r>0$. Let the function $g$ be defined by (3.2). We would like to find $\varrho_{0}>0$ such that $F(u)>0$ for all $\|u\|_{2}=\varrho_{0}$. By (4.1) this is satisfied provided $\varrho_{0}>0$ is such that $g\left(\varrho_{0}\right)=\alpha \varrho_{0}-\beta \varrho_{0}>\|b\|_{2}$. Using $\|b\|_{2}<\frac{2}{3} \lambda_{1} \sqrt{-4 \lambda_{1} /(3 h r)}=\frac{2}{3} \alpha \sqrt{\alpha /(3 \beta)}=g\left(\varrho_{m}\right)$, we put $\varrho_{0}=\varrho_{m}$. Thus, $F(u) \geqslant \min _{\|u\|=\varrho_{0}} F(u)>0$ for all $\|u\|_{2}=\varrho_{0}$.

The rest of the proof is similar to the proof of Theorem 3.5 if we interchange the role of the functional $G$ therein by $F$. 


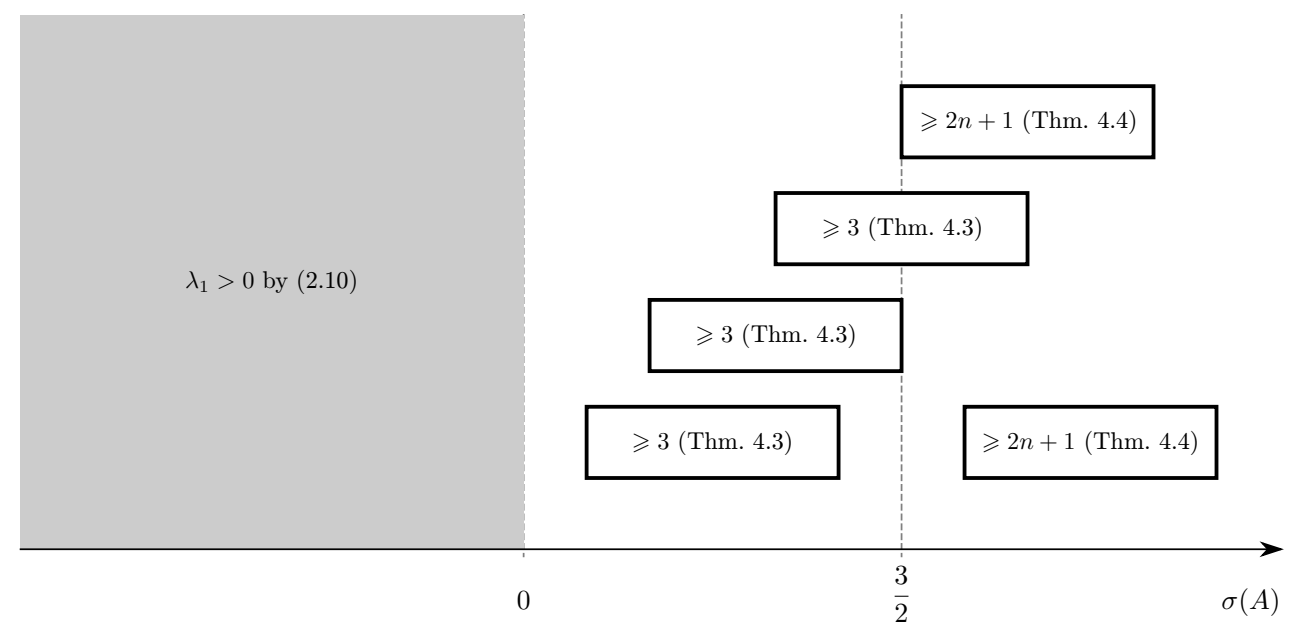

Figure 2. Dependence of number of solutions in the monostable case $r<0$ with positive diffusion based on the spectrum $\sigma(A)$ of the matrix $A=\frac{1}{2}(1-h r) I-\left(\frac{1}{2} h d / \mu^{2}\right) L$. The picture depicts various positions of $\lambda_{1}$ (the left end of the region) and $\lambda_{N}$ (the right end). Note, that (2.10) implies that the spectrum $\sigma(A)$ is always positive.

The following two theorems are consequences of Lemma 4.2 and can be shown analogously as Theorems 3.6 and 3.7.

Theorem 4.3. Let $r<0$ and $\|\varphi\|_{2}<\frac{2}{3} \lambda_{1} \sqrt{-4 \lambda_{1} /(3 h r)}$. Then at each time instant $n \in \mathbb{N}$ there exist at least three solutions of (2.2) which form trajectories $u^{n}$, $n \rightarrow \infty$.

Theorem 4.4. Let $r<0, \lambda_{1} \geqslant \frac{3}{2}$, and $\|\varphi\|_{2}<\frac{2}{3} \lambda_{1} \sqrt{-4 \lambda_{1} /(3 h r)}$. Then at each time instant $n \in \mathbb{N}$ there exist at least $2 n+1$ solutions of (2.2) which form trajectories $u^{n}, n \rightarrow \infty$.

The formula (2.10), Theorem 4.3, and Theorem 4.4 imply the following statement.

Corollary 4.5. Let $r<0$ and

$$
\|\varphi\|_{2}<\frac{1}{3 \mu^{2}}\left(\mu^{2}-h \mu^{2} r-h d \nu_{1}\right) \sqrt{\frac{-2}{3 h \mu^{2} r}\left(\mu^{2}-h \mu^{2} r-h d \nu_{1}\right)} .
$$

Then at each time instant $n \in \mathbb{N}$ there are at least three solutions of (2.2) which form trajectories $u^{n}, n \rightarrow \infty$. Moreover, if $r \leqslant-2 / h-d \nu_{1} / \mu^{2}$, then at each time instant $n \in \mathbb{N}_{0}$ there exist at least $2 n+1$ solutions of (2.2) which form trajectories $u^{n}, n \rightarrow \infty$. 
The results of this section and the consequent dependence of the number of solutions on the eigenvalues of the matrix $A=\frac{1}{2}(1-h r) I-\left(\frac{1}{2} h d / \mu^{2}\right) L$ and the reaction parameter $r$ are depicted in Figs. 2 and 3.
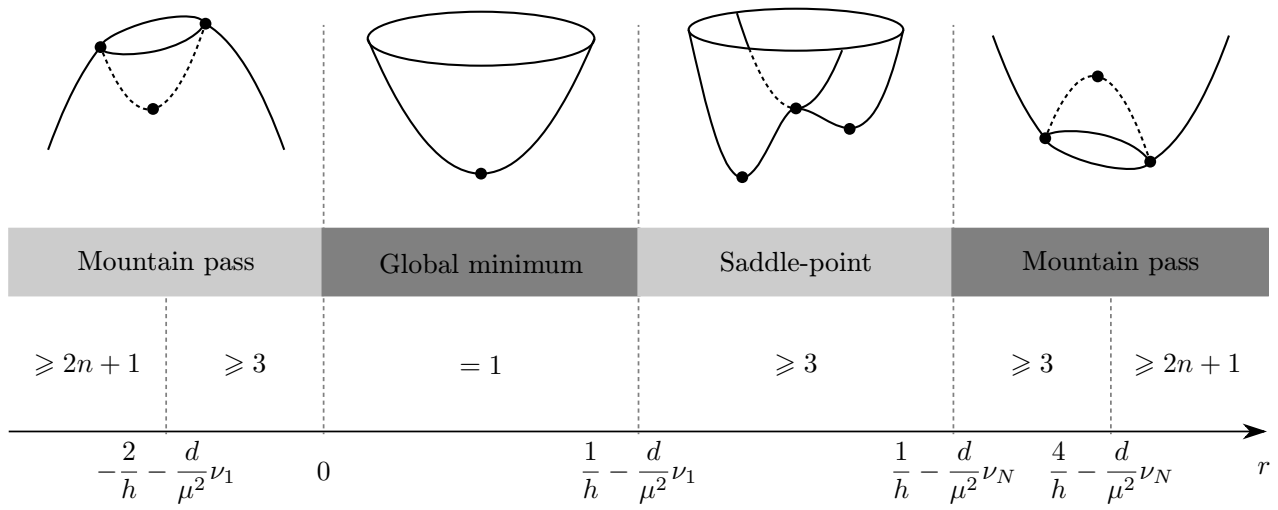

Figure 3. Dependendce of the geometry of $F$ defined in (2.9) and the number of solutions of (2.7) on the reaction parameter $r \in \mathbb{R}$. The picture summarizes the results from Section $3(r \geqslant 0)$ and from Section $4(r<0)$ for fixed $h, \mu, d>0$.

\section{DYNAMICS OF ONE POINT PROBLEM}

In order to illustrate the rich dynamics of the implicit discretization of lattice Nagumo equation, we describe in detail the infinite number of bounded trajectories of a reduced problem. We consider a one-point problem, i.e., the implicit discretization (2.2) with $N=1$ and uniform spatial discretization step $\mu=1$,

$$
\begin{cases}\frac{u_{1}^{n+1}-u_{1}^{n}}{h}=-2 d u_{1}^{n+1}+r u_{1}^{n+1}\left(1-\left(u_{1}^{n+1}\right)^{2}\right), & n \in \mathbb{N}_{0}, \\ u_{1}^{0}=\varphi_{1}, & n \in \mathbb{N}_{0} \\ u_{0}^{n}=0, & n \in \mathbb{N}_{0} . \\ u_{2}^{n}=0, & \end{cases}
$$

We will explore the dynamics in the parameter region with $d>0$ and $r<0$ but similar dynamics could be observed in other parameter regions with the mountain pass geometry, see Fig. 3 .

In each time step $n \in \mathbb{N}_{0}$, the problem (5.1) corresponds to the problem of finding $x=u_{1}^{n+1} \in \mathbb{R}$ which for given $b=u_{1}^{n}$ satisfies the equation

$$
\frac{x-b}{h}=-2 d x+r f(x)
$$


or, alternatively, $x(1+2 h d)-h r f(x)=b$. Our first goal is to describe the properties of the left-hand side. By rewriting it as a cubic function (see Fig. 4)

$$
\psi(x)=x\left(h r x^{2}+1+h(2 d-r)\right)
$$

we obtain trivial observations about its limit behavior and its roots.

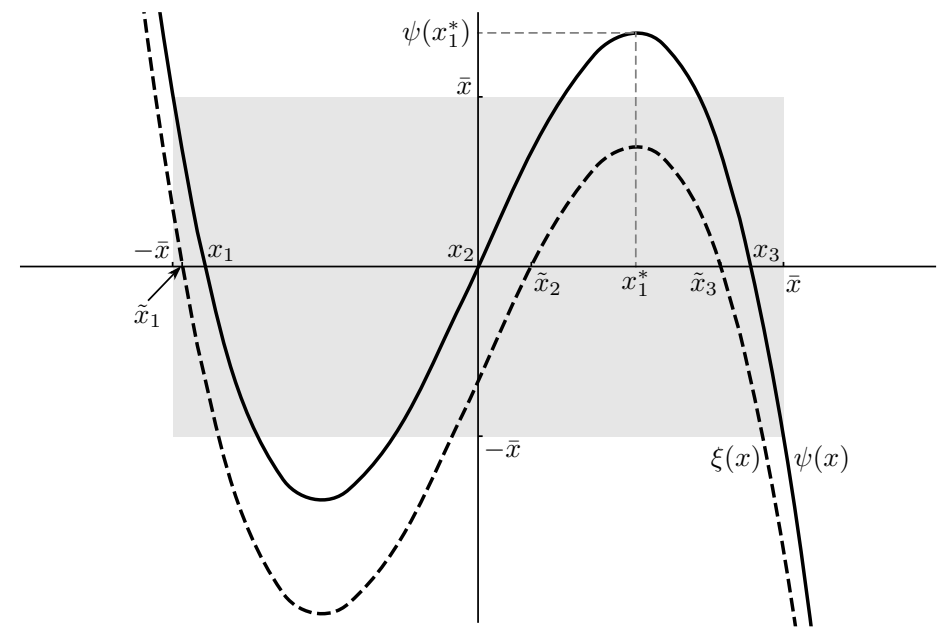

Figure 4. Illustration of quantities and functions from Section 5.

Lemma 5.1. Let $d>0$ and $r<0$. Then the function $\psi$ defined by (5.2) satisfies:

(1) $\psi( \pm \infty)=\mp \infty$,

(2) there exist three solutions of the equation $\psi(x)=0$,

$$
x_{2}=0, \quad x_{1,3}= \pm \sqrt{-\frac{1+h(2 d-r)}{h r}} .
$$

P r o of. The first statement follows immediately from the fact that $h r<0$. The roots can be obtained by solving the equation $\psi(x)=x\left(h r x^{2}+1+(2 d-r) h\right)=0$. Note that $x_{1,3}$ are well-defined since $h r<0$ and $1+h(2 d-r)>0$.

Similarly, we can easily find the stationary points of the function $\psi$.

Lemma 5.2. Let $d>0$ and $r<0$. Then the function $\psi$ defined by (5.2) has two stationary points

$$
x_{1,2}^{*}= \pm \sqrt{-\frac{1+h(2 d-r)}{3 h r}}=\sqrt{\frac{1}{3}} x_{1,3} .
$$


Pr o of. Differentiating (5.2), we obtain $\psi^{\prime}(x)=3 h r x^{2}+1+h(2 d-r)$. Setting $\psi^{\prime}(x)=0$ and rearranging the terms, we get (5.3).

In order to ensure that the solutions of the recurrence equation (5.1) remain bounded we introduce the quantity $\bar{x}>0$ such that $\psi(x)=-x$. Using the definition of $\psi(5.2)$, we obtain

$$
\bar{x}=\sqrt{\frac{-2-(2 d-r) h}{h r}} .
$$

Again, $\bar{x}$ is well-defined, since both $-2-(2 d-r) h<0$ and $h r<0$.

The key auxiliary statement compares the values of $\psi\left(x_{1}^{*}\right)$ and $\bar{x}$.

Lemma 5.3. Let $d>0, r<0$, and

$$
h>\bar{h}=\frac{2}{2 d-r} .
$$

Then $\psi\left(x_{1}^{*}\right)>\bar{x}$.

Pro of. First, we observe that

$$
\psi\left(x_{1}^{*}\right)=\frac{2}{3 \sqrt{3}} \sqrt{-\frac{(1+h(2 d-r))^{3}}{h r}} .
$$

Consequently, we can apply (5.4) to get

$$
\begin{aligned}
\frac{\psi\left(x_{1}^{*}\right)}{\bar{x}} & =\frac{\frac{2}{3 \sqrt{3}} \sqrt{-(1+h(2 d-r))^{3} /(h r)}}{\sqrt{-2-h(2 d-r) /(h r)}}=\frac{2}{3 \sqrt{3}} \sqrt{\frac{(1+h(2 d-r))^{3}}{2+h(2 d-r)}} \\
& =\frac{2}{3 \sqrt{3}} \sqrt{\frac{(-h(2 d-r)-1)^{3}}{-h(2 d-r)-2}} .
\end{aligned}
$$

Let us define an auxiliary function $\omega(\tau)$ by

$$
\omega(\tau)=\frac{(\tau-1)^{3}}{\tau-2}
$$

Since

$$
\omega^{\prime}(\tau)=2 \tau-1-\frac{1}{(\tau-2)^{2}},
$$

we observe that $\omega^{\prime}(\tau)<0$ for all $\tau<0$. Consequently, the function $\omega(\tau)$ is strictly decreasing for $\tau<0$. This, in turn, implies that $\frac{2}{3 \sqrt{3}} \sqrt{\omega(\tau)}$ is also strictly decreasing. 
Let us denote $\tau=-h(2 d-r)$, the assumption (A) implies that $\tau<-2$. Since $\omega(\tau)$ is decreasing for $\tau<-2$, we have

$$
\frac{2}{3 \sqrt{3}} \sqrt{\omega(\tau)}>\frac{2}{3 \sqrt{3}} \sqrt{\omega(-2)}=\frac{2}{3 \sqrt{3}} \sqrt{\frac{27}{4}}=1 .
$$

Hence, (5.5) implies that under the assumption (A) the inequality $\psi\left(x_{1}^{*}\right) / \bar{x}>1$ holds, which completes the proof.

At this stage we are ready to show the existence of three solutions of the equation $\psi(x)=b$.

Theorem 5.4. Let $d>0, r<0$, and let the assumption (A) be satisfied. Then for each $b \in[-\bar{x}, \bar{x}]$ there exist three distinct solutions $\tilde{x}_{1}, \tilde{x}_{2}, \tilde{x}_{3}$ of the equation $\psi(x)=b$. Moreover,

$$
\tilde{x}_{1}, \tilde{x}_{2}, \tilde{x}_{3} \in[-\bar{x}, \bar{x}] .
$$

P r o of. Without loss of generality, we can assume that $b>0$. First, note that Lemma 5.3 implies that $\psi\left(x_{1}^{*}\right)>\bar{x} \geqslant b$. Therefore, the function (see Fig. 4 )

$$
\xi(x)=\psi(x)-b
$$

attains a positive local maximum at $x_{1}^{*}$ and a negative local minimum at $x_{2}^{*}$. This implies that there exist three roots of $\xi(x)=0$ and they satisfy $\tilde{x}_{1} \in\left[-\bar{x}, x_{2}\right]$, $\tilde{x}_{2} \in\left[0, x_{1}^{*}\right]$, and $\tilde{x}_{3} \in\left[x_{1}^{*}, x_{3}\right]$. This completes the proof.

We emphasize that the assumption (A) not only ensures the existence of three solutions (in fact, this is true for any $h>0$ ) but more importantly the fact that the three solutions are uniformly bounded by $\bar{x}$. Consequently, we can extend the trajectories in the following way (see Fig. 5 for its illustration).

Corollary 5.5. Let $d>0, r<0, \varphi_{1} \in[-\bar{x}, \bar{x}]$, and let the assumption (A) be satisfied. Then at each time instant $n \in \mathbb{N}_{0}$ there exist $3^{n}$ solutions of (5.1) which form trajectories $u_{1}^{n}, n \rightarrow \infty$. Moreover, all the solutions are bounded and satisfy $u_{1}^{n} \in[-\bar{x}, \bar{x}]$ for all $n \in \mathbb{N}_{0}$.

Proof. Theorem 5.4 implies that for any initial condition $\varphi_{1} \in[-\bar{x}, \bar{x}]$ there exist three distinct ${ }^{k} u_{1}^{1}, k=1,2,3$, satisfying

$$
\psi\left(u_{1}^{1}\right)=u_{1}^{1}(1+2 d h)+h r f\left(u_{1}^{1}\right)=\varphi_{1} .
$$


To each of these three distinct values ${ }^{k} u_{1}^{1}$ we can apply Theorem 5.4 to get the existence of three distinct ${ }^{j} u_{1}^{2}, j=1,2,3$, satisfying

$$
\psi\left(u_{1}^{2}\right)=u_{1}^{2}(1+2 d h)+h r f\left(u_{1}^{2}\right)={ }^{k} u_{1}^{1} .
$$

This implies the existence of nine distinct pairs $\left({ }^{k} u_{1}^{1},{ }^{j} u_{1}^{2}\right)$. Straightforwardly, for each fixed $n \in \mathbb{N}$, we obtain the existence of $3^{n} n$-tuples $\left(u_{1}^{1}, u_{1}^{2}, \ldots, u_{1}^{n}\right)$ which satisfy $\operatorname{IBVP}(5.1)$ and $u_{1}^{m} \in[-\bar{x}, \bar{x}], m=1,2, \ldots, n$. Moreover, Lemma 2.2 implies that $3^{n}$ values of $u_{1}^{n}$ cannot coincide. The statement of the corollary follows immediately.

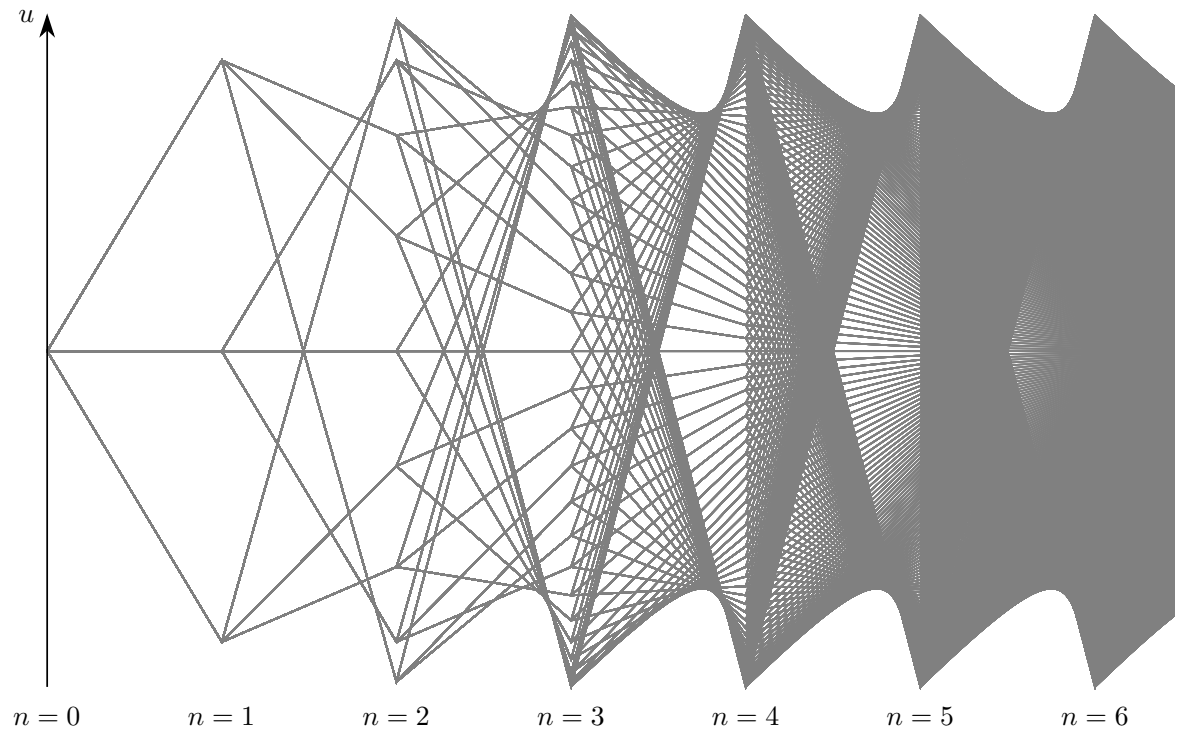

Figure 5. Illustration of Corollary 5.5. The picture shows $3^{6}=729$ trajectories of the first 6 iterations of the implicit one-point problem (5.1) with the initial condition $\varphi_{1}=0$.

\section{Negative Diffusion}

Motivated by [12] we consider here the case with negative diffusion parameter $d<0$. We claim that the reformulation of (2.2) into the algebraic problem (2.7) with the energy functional (2.8) (or (2.9)) remains the same and Lemmas 2.1 and 2.2 still hold.

We do not present a detailed analysis with all proofs, we focus only on three key structural differences. First, we will see that the problem (2.2) with $d<0$ need not have a global solution. Second, there exist values of $d<0$ for which any $r>0$ yields 
nonuniqueness. Finally, in the monostable case $r<0$ the problem with negative diffusion $d<0$ can have a unique solution.

Let us observe at the beginning that the definiteness of the matrix $\left(\frac{1}{2} h d / \mu^{2}\right) L$ changes for $d<0$. It is positive definite contrary to the case with positive diffusion $d>0$. Thus, if we preserve the notation $\lambda_{1}<\lambda_{2}<\ldots<\lambda_{N}$ as the strictly increasing sequence of eigenvalues of $A=\frac{1}{2}(1-h r) I-\left(\frac{1}{2} h d / \mu^{2}\right) L$, then (2.10) does not hold. However, one can show analogously that

$$
\lambda_{k}=\frac{1-h r}{2}-\frac{h d}{2 \mu^{2}} \nu_{N+1-k}, \quad k=1,2, \ldots, N .
$$

The corresponding system of orthogonal eigenvectors is

$$
\begin{aligned}
\phi_{k} & =\eta_{N+1-k} \\
& =\left(\sin \frac{(N+1-k) \pi}{N+1}, \sin \frac{2(N+1-k) \pi}{N+1}, \ldots, \sin \frac{N(N+1-k) \pi}{N+1}\right)^{\top},
\end{aligned}
$$

$k=1,2, \ldots, N$.

We observe the first key difference. One can see that in the case with positive diffusion, the problem (2.2) has always at least one global solution for all values of the reaction parameter $r \in \mathbb{R}$. That is not true with $d<0$. Let us consider the problem (2.2) with $d<0$ and without the reaction term (i.e., assume $r=0$ ). Hence, we want to analyze the linear implicit discrete problem

$$
\begin{cases}\frac{u_{i}^{n+1}-u_{i}^{n}}{h}=d \frac{u_{i-1}^{n+1}-2 u_{i}^{n+1}+u_{i+1}^{n+1}}{\mu^{2}}, & i \in\{1,2, \ldots, N\}, n \in \mathbb{N}_{0}, \\ u_{i}^{0}=\varphi_{i}, & i \in\{1,2, \ldots, N\}, \\ u_{0}^{n}=0, & n \in \mathbb{N}_{0}, \\ u_{N+1}^{n}=0, & n \in \mathbb{N}_{0},\end{cases}
$$

in which $d<0$. Again, let $n \in \mathbb{N}_{0}$ and $\left(u_{i}^{n}\right)_{i=0}^{N+1}$ be given. The problem to find a solution $\left(u_{i}^{n+1}\right)_{i=0}^{N+1}$ at $n+1$ is by (2.7) equivalent to the algebraic equation (using the notation (2.6))

$$
u=b+\frac{h d}{\mu^{2}} L u, \quad \text { or } \quad\left(I-\frac{h d}{\mu^{2}} L\right) u=b .
$$

However, recall that the matrix $L$ is negative definite. Thus, if $d<0$, it can happen that $I-\left(\frac{1}{2} h d / \mu^{2}\right) L$ (which is in fact the matrix $2 A$ with $A=\frac{1}{2}(1-h r) I-\left(\frac{1}{2} h d / \mu^{2}\right) L$ and $r=0$ ) has a zero eigenvalue. In such a case, i.e., if $\lambda_{k}=0$ or equivalently, $\nu_{N+1-k}=\mu^{2} /(h d)$ for some $k=1,2, \ldots, N$, the problem (6.4) has a solution (even 
infinitely many, the solution set is $\mathcal{S}=u_{0}+p \phi_{k}, p \in \mathbb{R}$, with $u_{0}$ such that $\left(u_{0}, \phi_{k}\right)=$ 0 ) if and only if $\left(b, \phi_{k}\right)=0$, i.e., $b$ is orthogonal to $\phi_{k}$ (by the Fredholm Alternative, see e.g. [9], Theorem 1.1.25).

Lemma 6.1. Let $d<0, r=0$, and $\lambda_{k}=0$ (or equivalently, $\nu_{N+1-k}=\mu^{2} /(h d)$ ) for some $k=1,2, \ldots, N$. Then there exists a unique solution $u$ of $(2.2)$ which exists for every $(n, i) \in \mathbb{N}_{0} \times\{0,1,2, \ldots, N, N+1\}$ if and only if $\left(\varphi, \phi_{k}\right)=0$.

P r o of. If there exists a unique global solution of (2.2) with $d, r, h$, and $\mu$ satisfying the assumptions, it is a solution of (6.3). Thus, there has to be a solution of (6.4) with $b=\varphi$. The Fredholm Alternative (see e.g. [9], Theorem 1.1.25 again) yields that $\left(\varphi, \phi_{k}\right)=0$.

Conversely, if $\left(\varphi, \phi_{k}\right)=0$, then there exists a continuum of solutions of (6.3) at $n=1$ which correspond to the solution set $\mathcal{S}=u_{0}+p \phi_{k}, p \in \mathbb{R}$, of (6.4) with $u_{0}$ such that $\left(u_{0}, \phi_{k}\right)=0$. However, only one of the solutions could be extended for higher values of $n$, since only $b=u_{0} \in \mathcal{S}$ satisfies again $\left(\varphi, \phi_{k}\right)=0$. One can continue by induction to obtain the statement.

Re mark 6.2. One can write the condition $\left(\varphi, \phi_{k}\right)=0$ thanks to (6.2) as

$$
\sum_{i=0}^{N} \varphi_{i} \sin \frac{i(N+1-k) \pi}{N+1}=0 .
$$

Lemma 6.3. Let $d<0, r=0$, and $\lambda_{k} \neq 0$ (or equivalently, $\nu_{N+1-k} \neq \mu^{2} /(h d)$ ) for all $k=1,2, \ldots, N$. Then there exists a unique solution $u$ of $(2.2)$ which exists for every $(n, i) \in \mathbb{N}_{0} \times\{0,1,2, \ldots, N, N+1\}$ for any $\varphi$.

Proof. Since the matrix $2 A=I-\left(\frac{1}{2} h d / \mu^{2}\right) L$ is invertible, the linear algebraic problem (6.4) has a unique solution. Therefore, at each time instant $n \in \mathbb{N}_{0}$ the uniquely determined $\left(u_{i}^{n}\right)_{i=0}^{N+1}$ implies the uniquely determined $\left(u_{i}^{n+1}\right)_{i=0}^{N+1}$ satisfying (6.3) (and thus (2.2)) which corresponds to the unique solution $u_{0}=$ $\left(I-\left(\frac{1}{2} h d / \mu^{2}\right) L\right)^{-1} b$ of $(6.4)$.

Since the case with $r=0$ has been discussed in detail now, we assume that $r \neq 0$ (i.e., the reaction term is always present) in the sequel of this section.

We present (without proofs) the existence and (non)uniqueness results for (2.2) with $d<0$ and $r>0$. Analogously as for $d>0$, we distinguish several cases of definiteness of $A=\frac{1}{2}(1-h r) I-\left(\frac{1}{2} h d / \mu^{2}\right) L$.

Let us start again with the positive (semi)definite matrix $A=\frac{1}{2}(1-h r) I-$ $\left(\frac{1}{2} h d / \mu^{2}\right) L$.

Theorem 6.4. Let $d<0, r\rangle 0$, and $\lambda_{1} \geqslant 0$. Then problem (2.2) has a unique solution $u$ and it exists for every $(n, i) \in \mathbb{N}_{0} \times\{0,1,2, \ldots, N, N+1\}$. 
The following statement is an immediate consequence of Theorem 6.4 and (6.1).

Corollary 6.5. Let $d<0$ and $0<r \leqslant 1 / h-d \nu_{N} / \mu^{2}$. Then problem (2.2) has a unique solution $u$ and it exists for every $(n, i) \in \mathbb{N}_{0} \times\{0,1,2, \ldots, N, N+1\}$.

The following two results state the nonuniqueness for (2.2) under the assumption that $A=\frac{1}{2}(1-h r) I-\left(\frac{1}{2} h d / \mu^{2}\right) L$ is negative definite, or indefinite (including negative definiteness).

Theorem 6.6. Let $d<0, r>0, \lambda_{N}<0$, and $\|\varphi\|_{2}<-\frac{2}{3} \lambda_{N} \sqrt{-4 \lambda_{N} /(3 h r)}$. Then at each time instant $n \in \mathbb{N}$ there exist at least three solutions of (2.2) which form trajectories $u^{n}, n \rightarrow \infty$. Moreover, if $\lambda_{N} \leqslant-\frac{3}{2}$, then at each time instant $n \in \mathbb{N}$ there exist at least $2 n+1$ solutions of (2.2) which form trajectories $u^{n}, n \rightarrow \infty$.

Again, the following corollary is an immediate consequence of Theorem 6.6 and (6.1).

Corollary 6.7. Let $d<0, r>0, r>1 / h-d \nu_{1} / \mu^{2}$, and

$$
\|\varphi\|_{2}<\frac{1}{3 \mu^{2}}\left(h \mu^{2} r-\mu^{2}+h d \nu_{1}\right) \sqrt{\frac{2}{3 h \mu^{2} r}\left(h \mu^{2} r-\mu^{2}+h d \nu_{1}\right)} .
$$

Then at each time instant $n \in \mathbb{N}$ there are at least three solutions of (2.2) which form trajectories $u^{n}, n \rightarrow \infty$. Moreover, if $r \geqslant 4 / h-d \nu_{1} / \mu^{2}$, then at each time instant $n \in \mathbb{N}$ there exist at least $2 n+1$ solutions of (2.2) which form trajectories $u^{n}, n \rightarrow \infty$.

Theorem 6.8. Let $d<0, r>0$, and $\lambda_{i}<\ldots<\lambda_{j}<0 \leqslant \lambda_{j+1}<\ldots<\lambda_{N}$. Then there exists $\delta>0$ such that for every $\varphi \in \mathbb{R}^{N}$ satisfying $\|\varphi\|_{2}<\delta$ there are at least three solutions of (2.2) at each time instant $n \in \mathbb{N}$ which form trajectories $u^{n}$, $n \rightarrow \infty$.

Let us emphasize that Theorem 6.4 hides the second key difference from the positive diffusion case. Let us focus on the analysis for which the values of parameters $A=\frac{1}{2}(1-h r) I-\left(\frac{1}{2} h d / \mu^{2}\right) L$ are positive (semi)definite. Since $r>0$, then the eigenvalues of $\frac{1}{2}(1-h r) I$ are at most $\frac{1}{2}$ for all values of $r>0$. Since $\left(\frac{1}{2} h d / \mu^{2}\right) L$ is positively definite, it can happen (contrary to the case of positive diffusion) that for sufficiently small values of $d<0(d \ll 0)$, we have $A=\frac{1}{2}(1-h r) I-\left(\frac{1}{2} h d / \mu^{2}\right) L$ is negative (semi)definite or indefinite for all values of $r>0$. Therefore, contrary to the positive diffusion case (in which for any $d>0$ there are values of $r>0$ for which there is a unique global solution of (2.2)), for $d<0$ sufficiently small, there are at least three global solutions to (2.2) for any $r>0$. 
At the end of this section we only summarize the results for the remaining case of (2.2) with negative diffusion $d<0$ and monostable reaction term $r<0$. The reader can proceed in the same way to analyze this remaining problem in detail.

Theorem 6.9. Let $d<0, r<0$, and $\lambda_{N} \leqslant 0$. Then problem (2.2) has a unique solution $u$ and it exists for every $(n, i) \in \mathbb{N}_{0} \times\{0,1,2, \ldots, N, N+1\}$.

Corollary 6.10. Let $d<0$ and $1 / h-d \nu_{1} / \mu^{2} \leqslant r<0$. Then problem (2.2) has a unique solution $u$ and it exists for every $(n, i) \in \mathbb{N}_{0} \times\{0,1,2, \ldots, N, N+1\}$.

Theorem 6.11. Let $d<0, r<0, \lambda_{1}>0$, and $\|\varphi\|_{2}<\frac{2}{3} \lambda_{1} \sqrt{-4 \lambda_{1} /(3 h r)}$. Then at each time instant $n \in \mathbb{N}$ there exist at least three solutions of (2.2) which form trajectories $u^{n}, n \rightarrow \infty$. Moreover, if $\lambda_{1} \geqslant \frac{3}{2}$, then at each time instant $n \in \mathbb{N}$ there exist at least $2 n+1$ solutions of (2.2) which form trajectories $u^{n}, n \rightarrow \infty$.

Corollary 6.12. Let $d<0, r<0, r<1 / h-d \nu_{N} / \mu^{2}$, and

$$
\|\varphi\|_{2}<\frac{1}{3 \mu^{2}}\left(\mu^{2}-h \mu^{2} r-h d \nu_{N}\right) \sqrt{-\frac{2}{3 h \mu^{2} r}\left(\mu^{2}-h \mu^{2} r-h d \nu_{N}\right)} .
$$

Then at each time instant $n \in \mathbb{N}$ there exist at least three solutions of (2.2) which form trajectories $u^{n}, n \rightarrow \infty$. Moreover, if $r \leqslant-2 / h-d \nu_{N} / \mu^{2}$, then at each time instant $n \in \mathbb{N}$ there exist at least $2 n+1$ solutions of (2.2) which form trajectories $u^{n}, n \rightarrow \infty$.

Theorem 6.13. Let $d<0, r<0$, and $\lambda_{i}<\ldots<\lambda_{j} \leqslant 0<\lambda_{j+1}<\ldots<\lambda_{N}$. Then there exists $\delta>0$ such that for every $\varphi \in \mathbb{R}^{N}$ satisfying $\|\varphi\|_{2}<\delta$ there exist at least three solutions of (2.2) at each time instant $n \in \mathbb{N}$ which form trajectories $u^{n}, n \rightarrow \infty$.

Consequently, in contrast to the case of positive diffusion $d>0$ we can get unique solutions for the monostable case $r<0$ as well.

A cknowledgements. The authors gratefully acknowledge discussions with Christian Pötzsche and Pavel Krejčí which led to the questions asked in this paper. They are also thankful for valuable suggestions of both referees and especially those of editor Tomáš Vejchodský. 


\section{References}

[1] G. Allaire, S. M. Kaber: Numerical Linear Algebra. Texts in Applied Mathematics 55, Springer, New York, 2008.

zbl MR doi

[2] L. J.S. Allen: Persistence, extinction, and critical patch number for island populations. J. Math. Biol. 24 (1987), 617-625.

zbl MR doi

[3] A. Ambrosetti, P. H. Rabinowitz: Dual variational methods in critical point theory and applications. J. Funct. Anal. 14 (1973), 349-381.

zbl MR doi

[4] D. G. Aronson, H.F. Weinberger: Nonlinear diffusion in population genetics, combustion, and nerve pulse propagation. Partial Differential Equations and Related Topics 1974. Lecture Notes in Mathematics 446, Springer, Berlin, 1975, pp. 5-49.

zbl MR doi

[5] S.-N. Chow, J. Mallet-Paret, W. Shen: Traveling waves in lattice dynamical systems. J. Differ. Equations 149 (1998), 248-291.

zbl MR doi

[6] S.-N. Chow, W.X. Shen: Dynamics in a discrete Nagumo equation: Spatial topological chaos. SIAM J. Appl. Math. 55 (1995), 1764-1781.

[7] L. O. Chua, L. Yang: Cellular neural networks: applications. IEEE Trans. Circuits Syst. 35 (1988), 1273-1290.

zbl MR doi

MR doi

[8] D. C. Clark: A variant of the Lusternik-Schnirelman theory. Indiana Univ. Math. J. 22 (1972), 65-74.

zbl MR doi

[9] P. Drábek, J. Milota: Methods of Nonlinear Analysis. Applications to Differential Equations. Birkhäuser Advanced Texts Basler Lehrbücher, Springer, Basel, 2013.

[10] P. C. Fife, J. B. McLeod: The approach of solutions of nonlinear diffusion equations to travelling front solutions. Arch. Ration. Mech. Anal. 65 (1977), 335-361.

[11] M. Galewski, J.Smejda: On variational methods for nonlinear difference equations. J. Comput. Appl. Math. 233 (2010), 2985-2993.

zbl MR doi

zbl MR doi

2] H. J. Hupkes, E.S. Van Vleck: Negative diffusion and traveling waves in high dimensional lattice systems. SIAM J. Math. Anal. 45 (2013), 1068-1135.

13] H. J. Hupkes, E.S. Van Vleck: Travelling waves for complete discretizations of reaction diffusion systems. J. Dyn. Differ. Equations 28 (2016), 955-1006.

zbl MR doi

14] J.P. Keener: Propagation and its failure in coupled systems of discrete excitable cells. SIAM J. Appl. Math. 47 (1987), 556-572.

zbl MR doi

15] J. Mallet-Paret: The global structure of traveling waves in spatially discrete dynamical systems. J. Dyn. Differ. Equations 11 (1999), 49-127.

zbl MR doi

zbl MR doi

[16] G. Molica Bisci, D. Repovš: On some variational algebraic problems. Adv. Nonlinear Anal. 2 (2013), 127-146.

zbl MR doi

[17] J. Nagumo, S. Arimoto, S. Yoshizawa: An active pulse transmission line simulating nerve axon. Proc. IRE 50 (1962), 2061-2070.

[18] J. Otta, P. Stehlik: Multiplicity of solutions for discrete problems with double-well potentials. Electron. J. Differ. Equ. 2013 (2013), 14 pages.

zbl MR

[19] C. Pötzsche: Geometric Theory of Discrete Nonautonomous Dynamical Systems. Lecture Notes in Mathematics 2002, Springer, Berlin, 2010.

[20] P. H. Rabinowitz: Some minimax theorems and applications to nonlinear partial differential equations. Nonlinear Analysis. Academic Press, New York, 1978, pp. 161-177.

[21] P. H. Rabinowitz: Minimax Methods in Critical Point Theory with Applications to Differential Equations. CBMS Regional Conference Series in Mathematics 65, American Mathematical Society, Providence, 1986.

[22] A. Slavik: Invariant regions for systems of lattice reaction-diffusion equations. J. Differ. Equations 263 (2017), 7601-7626.

zbl MR doi

zbl MR doi

zbl MR doi

zbl MR doi

[23] A.Slavik, P. Stehlik: Dynamic diffusion-type equations on discrete-space domains. J. Math. Anal. Appl. 427 (2015), 525-545. 
[24] P. Stehlik: Exponential number of stationary solutions for Nagumo equations on graphs. J. Math. Anal. Appl. 455 (2017), 1749-1764.

[25] P. Stehlik, J. Volek: Maximum principles for discrete and semidiscrete reaction-diffusion equation. Discrete Dyn. Nat. Soc. 2015 (2015), Article ID 791304, 13 pages.

[26] P. Stehlik, J. Volek: Variational methods and implicit discrete Nagumo equation. J. Math. Anal. Appl. 438 (2016), 643-656.

zbl MR doi

[27] J. Volek: Landesman-Lazer conditions for difference equations involving sublinear perturbations. J. Difference Equ. Appl. 22 (2016), 1698-1719.

[28] J. Volek: Multiple critical points of saddle geometry functionals. Nonlinear Anal., Theory Methods Appl., Ser. A, Theory Methods 170 (2018), 238-257.

[29] B. Zinner: Existence of traveling wavefront solutions for the discrete Nagumo equation. J. Differ. Equations 96 (1992), 1-27.

Authors' address: Petr Stehlik, Jonáš Volek, Department of Mathematics and New Technologies for the Information Society, University of West Bohemia, Univerzitní 8, 30100 Plzeň, Czech Republic, e-mail: pstehlik@kma.zcu.cz, volek1@ntis.zcu.cz. 